Revue internationale P.M.E.

Économie et gestion de la petite et moyenne entreprise

\title{
Les PME dans la nouvelle phase de régulation : enjeux productifs et territoriaux
}

\section{Jacques Perrat}

Volume 3, numéro 3-4, 1990

URI : https://id.erudit.org/iderudit/1007986ar

DOI : https://doi.org/10.7202/1007986ar

Aller au sommaire du numéro

Éditeur(s)

Presses de l’Université du Québec

ISSN

0776-5436 (imprimé)

1918-9699 (numérique)

Découvrir la revue

Citer cet article

Perrat, J. (1990). Les PME dans la nouvelle phase de régulation : enjeux productifs et territoriaux. Revue internationale P.M.E., 3(3-4), 367-388. https://doi.org/10.7202/1007986ar
Résumé de l'article

A travers leur changement de forme et leur repositionnement économique et social, les PME deviennent des outils adéquats pour une gestion plus efficace des gisements de ressources internes et externes au profit, essentiellement, des firmes les plus puissantes. Elles jouent par là un rôle clé dans le nouveau dispositif de régulation en émergence, ce que nous essayons de cerner ici en en mesurant les enjeux, les avancées et les limites, tant sous l'aspect productif que sous l'aspect territorial. 


\title{
Les PME dans la nouvelle phase de régulation : enjeux productifs et territoriaux
}

Jacques PERRAT*

ADEES

Rhône-Alpes

\begin{abstract}
RÉSUMÉ
A travers leur changement de forme et leur repositionnement économique et social, les PME deviennent des outils adéquats pour une gestion plus efficace des gisements de ressources internes et externes au profit, essentiellement, des firmes les plus puissantes. Elles jouent par là un rôle clé dans le nouveau dispositif de régulation en émergence, ce que nous essayons de cerner ici en en mesurant les enjeux, les avancées et les limites, tant sous l'aspect productif que sous l'aspect territorial.
\end{abstract}

\section{SUMMARY}

Through the change in their forms and through their economic and social relocation, Small and Medium Sized Firms are becoming adequate tools for a more efficient gestion of internal and external fields of resource, mainly to the advantage of Major Firms. They therefore play a key part in the new arising regulation process. This is what we are trying to analyse here while pointing out the stakes of such a movement, its progress and its limits, as much in the productive aspect as in the territorial one.

* Jacques Perrat (docteur $3^{\circ}$ cycle en économie de la production) dirige l'ADEES RhôneAlpes, centre d'études et de recherche sur l'économie régionale créé à Lyon en 1975 à l'initiative de la C.G.T. Il travaille sur les politiques régionales et locales, les liens Régions-Europe, les stratégies des firmes dans leurs rapports aux évolutions scientifiques et techniques et à l'espace. Membre de l'Association de Science Régionale de Langue Française, il a publié plusieurs articles, notamment dans la Revue d'Économie Régionale et Urbaine. Adresse : Association pour le Développement des Études Économiques et Sociales en Rhône-Alpes, 42 bis, rue Tronchet - 69006 Lyon, France. Premier manuscrit reçu, février 1990. 


\section{RESUMEN}

A través de su cambio de forma y de posicion economica y social, las Pequenas y Medias Empresas vienen a ser instrumentos apropriados para una gestion mas eficaz de os yacimientos de recursos tanto internos como externos, principalmente en provecho de las firmas mas potentes. Por lo tanto, desempenan un papel clave en el nuevo proceso de regulacion que esta emergiendo. Es lo que tratamos de analizar aqui poniendo de realce las puestas, los adelantamientos, los limites de tal movimiento tanto bajo el aspecto productivo como bajo el aspecto territorial. 


\section{Introduction}

La régulation d'un système socio-économique est le processus complexe par lequel un ensemble de conditions (institutions, procédures, normes, rapports entre les acteurs, etc.) assurent, face à des décisions et stratégies individuelles multiples et parfois contradictoires, à la fois le maintien de la cohérence, la reproduction et le développement des potentialités de ce système.

Nous voulons montrer ici ${ }^{1}$ que c'est dans le cadre des tentatives de mise en place d'une nouvelle phase de régulation qu'il convient d'apprécier la montée des PME ainsi que les nouveaux rapports qu'elles entretiennent tant avec les firmes plus importantes qu'avec des acteurs tels que les Collectivités Territoriales.

Il est incontestable que l'on assiste depuis plusieurs années à une transformation assez marquée du «paysage» des firmes :

- l'ancien paysage pouvait se caractériser par la présence de grandes firmes souvent multinationales, très identifiables par la taille de leurs établissements et par leur contrôle financier d'un certain nombre d'entreprises régionales et locales, mais aussi par la présence d'entreprises de taille moyenne et petite dont la situation variait entre la sous-traitance «captive» par rapport à de grands donneurs d'ordres et une autonomie assez complète sur des parts de marchés importantes (avec toutefois un système des prix largement dominé par les multinationales);

- le nouveau paysage est beaucoup moins «lisible»: il met en avant scène des PME de taille et surtout de formes des plus diverses; de la micro-entreprise (faisant [temporairement] entièrement corps avec son(ses) créateur(s)), aux entreprises de quelques dizaines, voire quelques centaines de salariés, structurées en «grappes» plus ou moins floues, elles fonctionnent en réseaux à géométrie variable dont l'écheveau des liens financiers, technologiques, productifs, informationnels, etc., est particulièrement difficile à démêler. Parallèlement, la présence visible des grandes firmes tend à s'estomper au fur et à mesure qu'elles évoluent elles aussi vers des formes plus floues, vers une structuration en réseaux vecteurs plus d'une «norme», d'une logique de firme multinationale que d'un contrôle financier classique.

En réalité, le débat sur l'importance et le devenir des différents types de firmes ne peut avancer que si l'on cesse de se focaliser sur une opposition de taille entre PME et grandes firmes et si l'on essaie d'identifier leurs nouvelles formes, leurs interrelations nouvelles entre elles et leur rôle respectif réel dans les processus socioéconomiques.

1 Ce texte a fait l'objet d'une communication au colloque de l'Association de Science Régionale de Langue Française, «La Science Régionale et l'État (Girondins et Montagnards)» qui s'est tenu à Bordeaux du 4 au 6 septembre 1989. Il a été largement remanié et complété en août 1990 . 
On ne peut en effet légitimement induire des constats précédents une quelconque dissolution des grandes firmes, ni une remise en cause de leur position dominante sur les systèmes productifs. D'abord parce que le mouvement de rachatabsorption des PME par ces firmes n'en a pas cessé pour autant; ensuite et surtout parce que l'adoption de la forme réseau par la fraction dominante du capital peut être considérée comme le moyen d'assurer une gestion plus intensive et plus flexible de ressources jusque là peu ou mal exploitées.

Il y a bien là, selon nous, maintien de la domination des grandes firmes, mais d'une façon plus indirecte et plus diffuse qu'auparavant : la PME devient aujourd'hui l'outil pour le capital d'une gestion à la fois plus rapprochée (en termes de proximité) et plus distante (en termes de contrôle) des gisements de ressources aussi bien internes qu'externes.

C'est ce rôle clé de la PME que nous voudrions cerner, d'abord en précisant la place de ces entreprises dans le repositionnement général des firmes par rapport à des facteurs aussi importants que le système productif, les procès de production, le capital, le rapport capital-travail, les ressources externes (I), pour essayer ensuite d'apprécier dans quelles limites, avec quelles contradictions et quels enjeux productifs et territoriaux, la forme PME s'inscrit dans le nouveau dispositif de régulation en émergence (II).

\section{Les PME dans le repositionnement général des firmes}

Il nous semble pouvoir établir un lien étroit entre nouveaux positionnements des PME, renouvellement de leur forme et montée de leur fonction d'intermédiation.

\subsection{Place dans le système productif : un accompagnement du recentrage des groupes}

Les groupes multinationaux, en même temps qu'ils poursuivent le repartage entre eux des créneaux de production et des parts de marchés, opèrent depuis quelques années un net recentrage sur ce qu'ils considèrent comme leurs «métiers». Un tel mouvement ouvre une série d'opportunités pour des PME pouvant se placer sur des productions, fonctions et services complémentaires à ces métiers et indispensables au maintien de certaines positions des groupes concernés (par exemple : complément d'une gamme de produits à l'exportation). Mais ces opportunités se conjugent, pour ces PME, avec une dépendance relativement forte par rapport à la stratégie d'un groupe (ou de plusieurs). Ce sont d'ailleurs le plus souvent les groupes qui sélectionnent les PME qui leur semblent les plus aptes à assurer ces complémentarités, voire qui externalisent de telles fonctions en incitant la création d'entreprises par leurs salariés sur ces créneaux. 
Un tel repositionnement n'est pas sans conséquences sur la physionomie du système productif lui-même puisqu'il renforce l'impact de la stratégie des groupes sur la configuration nationale des branches comme sur l'équilibre des sections productives.

\subsection{Place dans le processus de production : un partenariat exigeant}

Ce point est évidemment étroitement lié au précédent (liaison pouvant d'ailleurs être assurée par la notion de filière de production). La transformation des processus productifs sous l'effet conjugué des mutations technologiques et des exigences de rentabilité induit une redéfinition de la position stratégique de chacun de leurs différents stades, redéfinition entraînant elle-même un repositionnement des firmes.

Au stade central du processus, celui de la production, la recherche d'une productivité supérieure, combinée à la flexibilité et à la compression des coûts, amène les grandes firmes à s'adresser à des «spécialistes» extérieurs pour la réalisation de produits complexes, de petites séries, de prototypes, mais surtout à donner un nouveau contenu à leurs relations avec certains fournisseurs ou soustraitants chargés d'offrir des prestations de plus haut niveau concernant des pièces ou même des sous-ensembles complets, en moyennes ou longues séries (Lecler, 1989).

Cette situation, baptisée "partenariat», écarte toute analyse simpliste des liens PME/grandes firmes : une PME qui accède au rang de "partenaire» gagne à l'évidence une marge importante d'autonomie par rapport à sa situation antérieure parl'élargissement de son champ d'intervention sur les différents stades du processus productif (y compris, souvent, la conception) et par l'élargissement de sa clientèle (la rentabilisation des lourds investissements nécessaires l'amenant à travailler pour plusieurs donneurs d'ordres concurrents). Elle peut alors s'imposer comme interlocuteur obligé. D'un autre côté, il est non moins évident que la pratique des flux tendus conduit à une véritable intégration des sous-traitants et fournisseurs dans le processus de production des donneurs d'ordres; les «chartes» signées à ces occasions sont particulièrement contraignantes, surtout en termes de qualité et de délais, mais aussi de prix; et la sélection des «partenaires» est sévère et fréquemment réexaminée.

La même évolution peut s'observer concernant la maintenance, de plus en plus étroitement intégrée au processus productif et qui est souvent déléguée dans les mêmes condititons à des «entreprises extérieures». Mais ce qu'il nous semble surtout intéressant de noter c'est l'importance accrue prise aujourd'hui par les phases amont (conception) et aval (commercialisation) du processus productif, comme par l'activité plus «parallèle» de la logistique, et leur lien beaucoup plus fort qu'auparavant avec les phases centrales. Et les mêmes objectifs de productivité, flexibilité, réduction des coûts, amènent les groupes à externaliser ces fonctions sur des unités de petite taille, combinant spécificité de savoir-faire et grande adaptabilité aux conditions 
environnementales. On observe ainsi une véritable spécialisation de certaines PME sur ces phases :

- Beaucoup des PME qui se créent sur les technopôles, parcs d'affaires, pépinières, ont pour unique créneau d'activité l'innovation en matière de produits ou de procédés. Leurs initiateurs sont souvent issus d'un centre de recherche public, voire d'un groupe leur ayant cédé l'exploitation d'une idée ou d'un brevet. Sur de telles activités, l'autonomie de ces entreprises est pratiquement totale; elles éprouvent par contre les plus grandes difficultés sitôt qu'elles envisagent un passage aux phases d'industrialisation et de commercialisation, passage nécessitant des moyens hors de leur portée, sauf si elles entrent dans le réseau d'entreprise(s) plus puissante(s). En fait, la plupart de celles qui réussissent à se maintenir sont "condamnées», comme nous le verrons plus loin, à se recentrer en permanence sur les créneaux de la conception et de l'innovation, avec tous les risques que comporte cette dépendance par rapport aux autres stades et aux firmes qui en ont la maîtrise'2

- La même analyse peut s'appliquer à tout un ensemble de petites entreprises intervenant très en aval, en intermédiation avec les variations du marché et les besoins des clients et spécialisées dans les études, conseils, assistance à la commercialisation, à l'exportation, au «design», etc. Le rôle de ces PME souvent minuscules ${ }^{3}$ est très important, notamment en matière $\mathrm{d}^{\text {' }}$ «effet en retour» sur la production et même sur la conception (le client participant de plus en plus à la définition même du produit dans de nombreuses activités). Par contre, leur spécificité tend à réduire leur autonomie de positionnement et à les fragiliser dans un contexte de mondialisation des marchés.

- Enfin, les activités de logistique se sont, elles aussi, rapprochées du processus de production en même temps qu'elles offrent à des PME la possibilité de valoriser des compétences en matière de transport, d'approvisionnement/ distribution, de gestion des stocks, de conditionnement, etc. Là encore, les PME en question se retrouvent à la fois plus autonomes et plus dépendantes de la stratégie des groupes contrôlant le processus, et doivent surtout être capables de faire face à des contraintes financières particulièrement lourdes : dans les grandes entreprises, aujourd'hui, les stocks ne sont plus entreposés dans un local approprié mais se trouvent, soit chez les sous-traitants, soit en circulation dans leurs camions ou dans ceux des transporteurs, soit dans les remorques acculées contre des ouvertures permettant l'approvisionnement direct des lignes de fabrication ${ }^{4}$.

2 L'importance de ces PME doit être relativisée : en France, selon le SESSI, les groupes industriels de plus de 2000 salariés effectuent $80 \%$ des investissements de recherchedéveloppement et les entreprises de moins de 50 salariés seulement $8 \%$.

3 Selon les ASSEDIC, en Rhône-Alpes, 69,2\% des établissements recensés en 1988 dans les «services aux entreprises» occupaient moins de 5 salariés !

4 Entre Renault à Douai et Bertrand Faure à Somain - $17 \mathrm{~km}$ - six camions tournent toutes les $35 \mathrm{mn}$ pour livrer les sièges des R19. 


\subsection{Place relative au capital : la disjonction des réseaux}

Le phénomène qui nous semble le mieux à même d'éclairer cette question de l'autonomie-dépendance des PME, c'est la disjonction qui tend à s'établir entre la propriété du capital et le contrôle effectif des points clés du processus productif.

Le repositionnement constaté des PME sur les différents stades de ce processus productif s'inscrit en effet dans un mouvement qui permet aux grandes firmes de s'assurer une maîtrise sur les activités utiles à la mise en oeuvre de leur stratégie, tout en investissant un minimum de capital et là seulement où un contrôle plus direct se révèle indispensable. En cas de rupture des relations, le désengagement en est facilité d'autant.

Ce mouvement d'externalisation (l'internalisation ayant fait la preuve de ses limites en matière d'organisation et de coûts) transforme peuà peu les grands groupes en «firmes-réseaux multinationales» (Delapierre et Michalet, 1989), dont l'organigramme des entreprises contrôlées peut de moins en moins se limiter aux seules liaisons financières et tend à englober de multiples relations périphériques avec des PME, relations pour partie bien définies (charte de sous-traitance, accord avec un transporteur pour assurer l'alimentation en flux tendus, partenariat avec une société de marketing...) mais pour partie aussi beaucoup plus virtuelles, constituant autant de «positions d'attente» (transfert de technologie pour un nouveau produit ou procédé, prêt de locaux à une PME pour développer une innovation, aide au conseil au coup par coup) pouvant déboucher sur des relations plus suivies, sans forcément s'accompagner d'une prise de participation.

Cette disjonction des réseaux de contrôle financier et des réseaux productifs (au sens large) permet leur combinaison en des configurations très variées, d'autant que les PME s'adaptent à ces nouvelles contraintes en modifiant elles aussi leurs «contours»:

- $\quad$ soit en fusionnant pour réagir à la sélection des sous-traitants par les grands donneurs d'ordres : ainsi PRATIC, à Besançon, et ADEMVA, a Cluses, entreprises familiales de découpage-emboutissage travaillant à $50 \%$ pour l'automobile *ont anticipé la réduction du nombre de fournisseurs décidée par PSA et RENAULT.»

- Soit en constituant elles aussi des réseaux libres de toute motivation financière et centrés sur la coopération pour faire avancer la solution de certains problèmes techniques, ou pour présenter à un client une réponse complète à des questions touchant plusieurs domaines ou plusieurs stades productifs.

- Soit en bourgeonnant en de multiples filiales correspondant à autant d'activités nouvelles, certes parfois pour bénéficier de primes publiques ou empêcher la formation d'un comité d'entreprise, mais surtout pour conserver une flexibilité liée à cette petite taille et limiter les risques industriels, sociaux et financiers.

5 Cité par L'Usine Nouvelle le 1/6/89. 
Le problème majeur pour ces PME n'est donc pas tant la dépendance financière par rapport à une grande firme précise, que leurs conditions d'accès au capital en général. Pour tout développement sérieux (lancement d'une nouvelle fabrication, passage à une production en plus longue série, innovation productive) elles doivent faire appel à des financements extérieurs, tout en ayant beaucoup de difficulté à se faire ouvrir des crédits à moyen et long terme.

Par exemple, la structure de PME organisées «en grappe» autour d' un "mini-holding», M.S.I., qui occupe environ 1200 salariés en Ardèche dans 8 sociétés distinctes chargées chacune d'une activité bien précise, a fonctionné longtemps essentiellement avec des capitaux familiaux, français et monégasques, sans liens financiers avec les grands donneurs d'ordres de l'automobile pour lesquels travaillent et investissent ces entreprises (équipements lourds comme des grosses presses et des moules, mais aussi recherche-développement et formation). Mais elle a été contrainte, en 1988, de recourir au marché boursier (Second Marché de Lyon), avec le concours d' un pool bancaire. Aujourd' hui, le holding voit son capital passer à $40 \%$ dans le public (dont $12 \%$ au groupe PENHOUETO et une participation de la Lyonnaise de Banque dans une de ses filiales). ${ }^{6}$

D'où une palette très variée de situations où l'on retrouve tantôt les grandes banques et investisseurs institutionnels, tantôt les sociétés de capital-risque, tantôt une dilution du capital dans le public, tantôt les grands groupes industriels, commerciaux, fiannciers. Les PME peuvent ainsi utiliser la forme «grappe» pour combiner les différentes entrées de capitaux, les différencier selon la nature des activités concernées et selon le degré de contrainte qui en résulte, s'agissant de capital public à objectif premier de développement des PME, de capital financier attendant uniquement une rentabilité financière, ou de capital industriel et/ou commercial risquant de déboucher à plus ou moins long terme sur une intégration stratégique.

Par exemple, METRAVIB, créée d partir des travaux et dans les locaux de l'École Centrale de Lyon pour développer des applications dans le domaine des vibrations, a dû, pour prendre son essor, ouvrir son capital pour plus de $50 \%$ à des partenaires extérieurs, dont le groupe St-GOBAIN. Puis ont été créées plusieurs filiales spécialisées par fonction et produit, sur lesquelles ont pu s'opérer des branchements de divers réseaux financiers. Ainsi, VECTABIB (production industrielle de microcapteurs pour l' automobile) a vue l' entrée à son capital d' INNOLION (CREDIT LYONNAIS), de PARIBAS et d' une société anglaise de "venture capital», $B B H Q$; (de même, l'activité de surveillance a été cédée à FRAMATOME, tandis qu'une nouvelle société, METRAVIB FLUIDES, a été créée pour développer l'activité «détection de fuites». Par contre, la société «mère» peut continuer de fonctionner comme un incubateur d' innovations, ce qui était sa vocation de départ.'

6 L'ADEES Rhône-Alpes travaille actuellement sur les relations partenaires-donneurs d'ordres à partir de l'exemple MECELEC-RVI.

7 L'ADEES R.A. suit particulièrement les trajectoires de ces PME innovantes et en rend compte dans ses publications. 
Mais pour tenter d'échapper à ces contraintes, les PME cherchent surtout à préserver au maximum leurs capacités d'autofinancement, d'où une pression constante sur leurs conditions internes de fonctionnement, le réinvestissement des bénéfices, le recours accru à une contribution des salariés (cette entrée du «capital salarial» n'étant évidemment pas sans conséquences sur l'évolution rapport capitaltravail).

\subsection{Place relative au rapport capital-travall : une mobilisation élargie des ressources humaines}

Un chercheur du GLYSI, B. Ganne (1988), a étudié une douzaine de PME de la région d'Annonay en Ardèche, région sur laquelle il s'était déjà penché il y a une dizaine d'années. A part deux créations au sens strict du terme, il a observé un grand nombre de reprises après difficultés traduites par de sévères suppressions d'emplois (de 40 à $50 \%$ ) et surtout un profond renouvellement de la physionomie de ces entreprises qui lui font parler d'un phénomène massif (7 cas sur 10) de «recréation». Et cette mutation porte moins sur les méthodes de production sur les innovations technologiques que sur la mise en oeuvre d'un nouveau «modèle social»: pour leur financement, ces entreprises se sont tournées vers les aides publiques, notamment régionales, départementales et locales, mais surtout vers l'implication de leurs salariés et leur participation directe soit au capital, soit au montage financier de relance, avec utilisation des indemnités de licenciement et de chômage, «avec le double avantage d'échapper par ce biais aux conditions draconiennes du marché tout en favorisant une forte intégration sociale dans l'entreprise».

Cet intéressement financier direct, mais aussi les différents systèmes de responsabilisation mis en place (affichage d'indices, réunions d'informations, cercles de qualités, définitions d'objectifs individuels à atteindre), ont pour effet, outre une «marginalisation» des structures de représentations syndicales, une forte adhésion des salariés aux objectifs de l'entreprise qui se traduit le plus souvent par la décision de réinvestir les profits, par exemple d'acheter une nouvelle machine, "pour tenir face à la concurrence» plutôt que de distribuer des bénéfices.

Une autre étude du GLYSI apporte un bon éclairage sur cet aspect de la question. M. F. Raveyre (1988) a fait porter sa réflexion sur un échantillon de PME beaucoup plus large (40 entreprises), couvrant tout le territoire national, et à partir d'un objectif tout différent puisqu'il s'agissait d'observer les entreprises entretenant des relations avec St-Gobain Développement (filiale du groupe S.G. spécialisée dans l'aide aux PME). Or, là encore, il se dégage de cetéchantillon «une forte homogénéité faisant apparaître un type de PME éloigné des modèles repérés dans les travaux antérieurs».

Dans ces PME, «si les dirigeants enquêtés possèdent majoritairement le capital de l'entreprise, celui-ci n'a plus le même statut»: cette détention du capital est 
considérée comme une garantie d'autonomie, mais dans la majorité des cas, il y a partage avec d'autres «associés-créateurs» et «direction collégiale» $(70,9 \%$ des entreprises ont mis en oeuvre ce modèle à un moment ou à un autre). Le capital n'est ainsi pas tant considéré comme un patrimoine personnel que comme «un moyen nécessaire pour que l'entreprise puisse fonctionner».

Cette «conception instrumentaliste du capital», renforcée par le fait que, pour le createur, le capital a d'abord permis de créer son propre emploi (et parfois celui de son(sa) conjoint(e)), tend à s'imposer de fait à l'ensemble des salariés et induit un type très particulier de rapport capital-travail qui nous parât être en lien étroit avec la position elle aussi particulière de ces $P M E$ dans le processus productif. M.F. Raveyre montre, en effet, que si plus de $64 \%$ d'entre elles sont spécialisées dans les productions unitaires et les petites séries ( $25 \%$ dans les moyennes séries et seulement $10 \%$ dans les grandes séries) et se présentent comme des «spécialistes du mouton à cinq pattes», tirant leurs performances de critères d'adaptation aux besoins des clients, de qualité, de maîtrise de problèmes complexes, cela tient moins au recours à des technologies sophistiquées («l'automatisation n'est pas recherchée systématiquement») qu'à une organisation de la production et du travail faisant appel à «la mobilisation des salariés, clef de voûte de la flexibilité et de la qualité».

Une telle mobilisation s'inscrit dans «une représentation dynamique de l'entreprise» fondée sur «le modèle de l'équipe» engagée dans une «aventure collective», qui estompe la relation salariale patron-employé et marginalise, là encore, même quand le patron est un ancien syndicaliste, les structures habituelles de représentation du personnel. Il peut alors exister une forte adéquation entre les performances productives demandées et une organisation du travail qui combine polyvalence des compétences, flexibilité des tâches selon le type de problème et l'urgence du moment, élasticité du temps de travail (travail plus tard le soir, travail le week-end, avec non pas rémunération d'heures supplémentaires mais récupération à d'autres moments selon les convenances personnelles). L'intégration des salariés est donc ici particulièrement forte : «un système de règles implicites est ainsi établi entre dirigeants et salariés. C'est dans la mesure où celui-ci peut fonctionner que le modèle de «l'équipe» trouve son fondement (et inversement c'est dans la mesure où l'on adhère à cet idéal qu'un tel système de pratiques est possible).»

Mais si ce type de rapport capital-travail est porteur, pour ces entreprises, de capacités certaines à occuper une place qualifiante dans le processus productif, il peut jouèr en même temps comme facteur hautement contraignant vis-à-vis de tout changement souhaité de position. C'est ce qui ressort notamment des observations effectuées sur des entreprises semblables au Danemark (Monsted, 1988): «L'organisation décentralisée d'une production fondée sur un travail de métier» met en oeuvre des qualifications «qui ne sont pas celles nécessaires au passage d'une production artisanale de prototypes à la production industrielle de masse». Ainsi, une PME ayant réussi une innovation de produit et qui voudrait se lancer dans la production massive devrait surmonter de grandes difficultés, les changements 
organisationnels à opérer étant autrement plus profonds que les modifications techniques nécessaires : «Un niveau élevé de qualification technique et une diversification des tâches où les travailleurs peuvent se servir de toute la gamme de leurs savoirs peuvent être bénéfiques à l'innovation de produits et à la résolution de problèmes. Mais ils conviennent moins bien à des activités industrielles répétitives où, qui plus est, leur coût est trop élevé.» Par ailleurs, «une production de masse avec un recours à une main-d'oeuvre non qualifiée exige de nouvelles modalités de contrôle» et donc pratiquement une remise en cause de ce modèle d'entreprise.

L'intérêt de ces différentes études, qui se recoupent d'ailleurs avec bien d'autres, est de montrer :

- que la mutation des PME dans leur forme, leur structure organisationnelle, leur mode de fonctionnement, n'est pas un épi-phénomène;

- qu'elle s'opère dans le sens de ce que l'on peut appeler «l'innovation sociale», plus que dans celui de l'innovation technologique, mais elle demeure en relation étroite avec la place occupée par l'entreprise dans le processus de production et, plus globalement, dans le système productif. De telles observations ne sauraient occulter, en effet, la situation beaucoup plus traditionnelle d'un grand nombre de PME à qui sont attribuées des places nettement moins valorisantes. Nous avons personnellement visité en Écosse une PME, implantée dans un "parc d'entreprise» flambant neuf construit par une collectivité locale et employant, dans des conditions de travail et d'hygiène-sécurité (et sans doute de salaire) déplorables, une main-d'oeuvre déqualifiée pour des opérations d'assemblage de cartonnages incluant les capots inférieurs d'ordinateurs pour alimenter en flux tendus, à quelques dizaines de kilomètres de là, les lignes robotisées d'une usine d'IBM...

\subsection{Place relative aux ressources externes : le relais obligé des Collectivités Territoriales}

Le repositionnement des firmes entre elles s'inscrit dans un processus plus large de repositionnement des firmes par rapport à leur environnement et par rapport à l'ensemble des ressources externes. Cela concerne autant le recentrage des grands groupes sur leurs métiers qui les amène, nous l'avons dit, à déléguer les compétences complémentaires nécessaires à tous un ensemble d' «entreprises extérieures» et de «partenaires», que les PME qui recherchent, au moins pour les plus dynamiques d'entre elles, un partage des tâches, une combinaison de compétences dans leurs relations avec d'autres $P M E$ partenaires «de second rang» et dans leurs relations avec les diverses ressources nécessaires à leur activité (recherche, innovation, formation, services, financement).

Un chercheur danois(Christensen, 1988) propose, pouréclairerces processus, les notions de «flux externes de compétences» et «qualifications croisées», en précisant que «les qualifications constituant le noyau interne [de compétences] n'ont 
aucune valeur sans la combinaison avec les flux externes de compétences. [...] La question de la gestion des ressources internes doit désormais inclure la gestion des ressources dans leur environnement. [...] La firme doit être conçue comme un système ouvert dans lequel les ressources externes sont mises en oeuvre en fonction des besoins de l'entreprise.»

Plus précisément, il nous semble que les positions nouvelles des PME les conduisent à exercer véritablement une fonction d'intermédiation entre les grandes firmes et les innovations, les marchés et plus largement ce que nous appelons des «gisements d'externalités» (Perrat, 1988).

Or le caractère intrinsèquement limité de la forme PME demande, pour répondre à cette exigence d'intermédiation, qu'interviennent d'autres acteurs, que soient trouvés des «relais», notamment au niveau des institutions. Et c'est dans cette perspective que se positionnent, depuis plusieurs années, les Collectivités Territoriales, en recentrant leurs politiques sur l'assistance tous azimuts aux PME pour leur faciliter l'accès à l'information scientifique et technique, les liens avec les établissements de recherche et de formation comme avec les organismes de transfert de technologies, la pénétration des marchés nationaux et internationaux, et surtout réaliser pour elles les montages financiers correspondants. Les Collectivités Territoriales interviennent aujourd'hui de plus en plus dans les opérations de création d'entreprise, de transmission-reprise, d'innovation en matière de procédés ou de produits, avec la particularité que moins la rentabilité est assurée, plus le risque est élevé et plus on observe un recours au type de capital qu'elles mobilisent : apports de fonds à long terme et à taux réduit, avec ou sans participation au capital, garantie et contre-garantie d'emprunts, montages d'opérations de capital-risque, surtout pour les démarrages d'entreprises, phase la moins couverte même par les sociétés de capital-risque mises en place par les grands investisseurs, ceux-ci ayant tendance «à minimiser les risques et optimiser les profits $\rangle^{8}$

Une telle politique s'alimente d'ailleurs périodiquement à des études faisant la preuve de l'inaptitude de la grande majorité des PME à assumer seules de telles transformations, à «assimiler la norme internationale», et de la nécessité de leur faire «franchir des seuils». Et ces interventions sont largement facilitées par le type particulier de rapport que les PME en question entretiennent avec le capital : c'est dans la mesure où il y $\mathrm{a}$ en quelque sorte externalisation d'un type spécifique de rapport capital-travail, qui estompe ses dimensions conflictuelles et met en avant la réalisation d'un «projet commun», que sont levés de façon souvent très consensuelle les obstacles à l'accès de ces entreprises aux ressources matérielles, humaines et financières. Les Collectivités Territoriales peuvent apporter ainsi tout «naturellement»

8 Les Échos du 24/5/89. De même, un article du Progrès (13/6/89) indiquait que sur les 30 000 entreprises dev ant changer de propriétaire dans les 10 ans à venir, seules les 1000 plus importantes intéresseraient les investisseurs qui considèrent ces entreprises comme «outils de spéculation financière» et recherchent «des plus-values élevées», «une rotation rapide du capital» et «une rentabilité à court et moyen terme». 
leur soutien à des opérations dont la distance semble grande avec les stratégies du capital dominant et où n'apparaît que la nécessité d'un renfort à la fonction instrumentale du capital.

Cela n'empêche pas les firmes multinationales de bénéficier parfois, «dans la foulée» pourrait-on dire, d'engagements directs et importants de ces Collectivités : par exemple, St-Gobain a obtenu pour sa nouvelle usine de verre plat de Salaize sur Sanne, dans l'Isère, outre des conditions d' implantation particulièrement avantageuses du Département et de la Commune, une subvention régionale de plus de 1 MF au titre de la formation du personnel. ${ }^{9}$ Plus généralement, les institutions financières chargées du développement régional, comme les S.D.R., apportent fréquemment leur aide aux filiales des grands groupes en les considérant sous leur «forme PME»...

Certes, les Collectivités Territoriales ne sont pas les seuls intervenants en la matière; l'État y joue un rôle très important ainsi que, de plus en plus, les institutions européennes. Par ailleurs, ces politiques ne sont pas toujours couronnées de succès. Les interventions des Régions, des Départements et des Villes manquent souvent de coordination et ne touchent habituellement que les PME les plus dynamiques, lesquelles, d'ailleurs, considèrent souvent ces interventions comme insuffisantes.

Il n'en reste pas moins que l'entrée en force des Collectivités Territoriales surce terrain est bien un des déterminants essentiels du mouvement de décentralisation amorcé au début des années 1980 , signifiant par là que le pilotage de processus socioéconomique de plus en plus complexes et leur ajustement dans le temps et dans l'espace ne peuvent plus s'obtenir efficacement au moyen d'un centre unique et étatique.

\section{PME et régulation : portée et limites d'un renouvel- lement}

Le repositionnement des PME, comme le renouvellement de leur forme, contribuent assez efficacement, nous semble-t-il, à la mise en oeuvre d'une nouvelle régulation socio-économique. Celle-ci est centrée sur une exploitation plus intensive, au plus près et «juste à temps» des ressources internes et externes, matérielles, humaines et financières. Mais chacune des avancées dans cette direction est un processus contradictoire; chacune suscite des contre-tendances et se heurte à des contraintes qui en limitent la portée tant au niveau productif qu'au niveau territorial.

9 Recueil des Actes Administratifs de la Région Rhône-Alpes, 1988-10. 


\subsection{Les PME dans l'enjeu productif : flexibilité, créativité et nouvelles exigences de productivité}

La complexité de l'approche de ces phénomènes provient de ce que les mêmes conditions qui font la force des PME en font en même temps la faiblesse. Il en est particulièrement ainsi de leur rapport au temps et surtout de leur capacité à gérer le rapport capital-travail.

\section{a) Un nouveau rapport au temps}

Aujourd'hui, «les schémas de production à technique «séparée» (permettant une dissociation spatiale et économique poussée entre la production des techniques et la production tout court) font place à des schémas à technologie «liée» ou «intégrée», impliquant une autoproduction évolutive des outils, ainsi que des rétroactions fortes et permanentes en marchés, produits et techniques» (Beckouche, Savy, Veltz, 1988). Les PME sont effectivement, nous l'avons vu, porteuses de réponses adaptées à ces nouveaux impératifs de souplesse et de flexibilité, à ces exigences d'adaptabilité permanente des ressources internes et de rétroactivité dans les plus brefs délais entre innovations de produits et de procédés et évolutions de la demande. Cette gestion du temps touche même les bâtiments de production : une PME fabriquant des sièges de voiture a implanté en Bretagne une usine dont l'architecture répond aux besoins de flexibilité d' une "chaîne de fabrication qui $s^{\prime}$ agrandit ou se réduit au gré des commandes». ${ }^{10}$

Mais les limites à cette réorganisation tiennent d'abord au même facteur qui assure aux PME cette flexibilité dans le temps : leur taille, c'est-à-dire leurs capacités en termes quantitatifs. Le capital, même baptisé «capital-risque», ne s'investit que dans les projets jugés suffisamment «porteurs»; la «surface» d'une entreprise en étant un élément décisif d'appréciation. Et surtout, dans de nombreux domaines «où les générations de produits se succèdent rapidement, la concentration des ressources en recherche-développement est un atout majeur»."

La dépendance de ces PME par rapport aux mouvements dans le temps du capital dominant est surtout flagrante du fait qu'aucune d'entre elles n'est à même d'assurer seule le processus de rétroaction marché-innovation dont la maîtrise d'ensemble ne peut guère appartenir qu'aux grandes «firmes-réseaux». Mais cela est valable aussi pour les phases productives au sens strict. Si à l'intérieur même d'une entreprise la gestion au plus juste du temps productif «actif», source d'élimination des rigidités, des temps morts dus à l'accumulation des opérations «passives», ne s'obtient qu'au prix d'un décloisonnement et d'une intégration des différentes fonctions, on voit mal comment un tel processus pourrait sans contradictions

10 Il s'agit de l'entreprise Treves à Ploërmel.

11 Relevé par L'Usine Nouvelle du 20/4/89 à propos du rachat d'Apollo par HewlettPackard. 
s'accommoder du fractionnement induit par le report sur des PME distinctes de ces mêmes fonctions. Un fonctionnement acceptable de l'ensemble ne peut donc aller de paire qu'avec l'exercice par les firmes «têtes de réseaux» d'une tutelle, certes indirecte, mais néanmoins étroite, sur ces PME, faisant peser de lourdes incertitudes sur l'établissement de leur «plan de carrière» à long et même à moyen terme; ce qui ne va pas forcément dans le sens de la plus grande efficacité.

Enfin, une limite non moins importante tient au fait que beaucoup de PME se retrouvent plus ou moins «captives» sur les positions gagnées et n'ont pratiquement d'autres ressources que le recours au mouvement perpétuel vers l'innovation productive et vers l'autoproduction de nouvelles entités au sein de structures en grappe ou en réseau, mouvement comportant à l'évidence de grands risques de fragilisation. Les dirigeants-fondateurs d' une PME de biotechnologies de LyonGerland nous ont ainsi confié que leurs actionnaires souhaitaient une évolution vers une plus grande stabilité par la définition de produits capables d' ancrer l'entreprise sur un ou des marchés précis ${ }^{12}$. Qu'il s'agisse de PME spécialisées dans la soustraitance ou de PME exploitant un nouveau créneau en termes de produit ou de procédé, leur pérennité et leur autonomie ne s'assurent qu'en étant constamment remises en question, puisque fondées sur leur capacité à maîtriser un savoir-faire évolutif ou à accompagner le plus longtemps possible une innovation sur la voie de l'industrialisation et de la commercialisation.

Nous décelons ici un enjeu considérable, celui de la conciliation à réaliser entre la mobilisation de nouveaux gisements de flexibilité et de créativité et la relative stabilité nécessaire au mûrissement des savoirs et des savoir-faire, à la construction de synergies et de cohérences, mais aussi à la diffusion dans l'ensemble du système productif des avancées réalisées notamment en termes de rapport capital-travail.

\section{b) Les prémisses d'une mobilisation supérieure des salariés}

Le rapport capital-travail joue un rôle particulièrement important en matière de régulation. Il détermine les articulations production-répartition et accumulationconsommation : le rapport salarial peut être considéré comme «forme institutionnelle» (ou plutôt «structurelle») essentielle puisque «caractérisant un type d'appropriation particulier du surplus» (Boyer, 1985; Le Bas, 1987). Mais aussi et surtout, il détermine le type de progression de la productivité, facteur décisif de la reproduction élargie d'un système socio-économique (Aglietta et Brender, 1984; Bellet, 1987).

Or les évolutions observées précédemment tendent à positionner un certain nombre de PME sur les activités ou fonctions où la mise en oeuvre de la force de travail est la plus exigente, en termes de mobilisation des savoirs et des savoir-faire, d'implication et de responsabilisation, sources les plus efficaces aujourd'hui d'une

12 Visite effectuée par H. Piquet-Gauthier avec qui nous coopérons pour son Mémoire de DEA en Économie de la Production (Université Lumière Lyon 2, sous la direction d'Y. Bouchut) sur le thème des «PME innovantes». 
nouvelle élévation de la productivité. En même temps, c'est précisément dans ces mêmes PME, nous l'avons vu, que l'aspect «rapport social» du capital est le plus facilement occulté par son aspect «facteur opératoire».

Ce rapport au capital sous sa seule forme «instrumentale» est d'ailleurs également développé au sein des grands groupes : ceux-ci tendent à apparaître comme «de vastes outils de gestion», comme des «agences» d'organisation contractuelles entre propriétaires et dirigeants, prêteurs et emprunteurs, employeurs et salariés, groupe et autres partenaires, et cherchant avant tout à optimiser le fonctionnement et les coûts de ces relations (De Montmorillon, 1989). Cette «théorie de l'agence» s'applique évidemment encore mieux à une structure de «firme-réseau».

La principale limite à l'aptitude régulatoire de cette transformation tient selon nous à sa portée restreinte par rapport à l'ampleur de l'enjeu productif. Cette mobilisation supérieure des salariés est réalisée de manière très sélective et partielle :

- quant aux unités de travail concernées : même sil'évolution des PME en ce sens est un phénomène assez large, et même si les grandes entreprises tentent souvent d'obtenir le même résultat en fractionnant et réorganisant leurs cellules de production, de larges fractions des unités productives restent encore à l'écart d'un tel mouvement.

- quant au champ de la «responsabilisation salariale»: l'intervention des salariés s'exerce rarement au-delà du champ de l'activité productive, la Direction se réservant de trancher les questions stratégiquement les plus importantes. Par ailleurs, tout le personnel n'est pas forcément impliqué ni dans une participation au capital, nià ce nouveau rapport à l'entreprise. En général, le degré d'une telle implication est fonction de la position sur la grille des classifications.

Or les exigences actuelles en matìre d'interdépendance des activités, des fonctions, des entreprises et des hommes ne peuvent se satisfaire durablement du caractère partiel et fractionné (et fractionnant, en termes de système productif!) de cette régulation. Elles appellent au contraire une valorisation de la force de travail individuelle et collective beaucoup plus large, se diffusant dans l'ensemble du processus de production, et tirant parti en même temps qu'elle les renforce des cohérences du système productif.

\subsection{Les PME dans l'enjeu territorial : logique de réseaux et ges- tion des Biens Territorlaux}

Depuis l'accentuation de leurs mouvements de restructuration et de redéploiement, les grandes firmes ont fait l'expérience des problèmes causés par une présence trop directe et trop massive dans certaines zones du territoire. Elles se sont également rendu compte qu'elles n'étaient pas les acteurs les plus performants pour une exploitation au plus près et ajustée dans le temps des ressources externes localisées. Ces deux raisons les ont conduites à compter de plus en plus sur des PME 
pour bénéficier d'une intermédiation dans l'accès à ces ressources. La politique des Collectivités Territoriales de soutien à la création et au développement des PME est venue renforcer le rôle de cette forme d'entreprise dans cet aspect déterminant du processus de régulation qu'est le rapport du système socio-économique à l'espace.

Les PME qui entrent dans ce mouvement représentent donc, notamment du point de vue de la grande firme, une fraction de l'ensemble des firmes assimilables à des «biens collectifs», au même titre que les autres ressources externes inscrites dans les territoires avec lesquels elles «font corps» (en langage marxiste nous pourrions dire qu'une fraction du capital devient ainsi assimilable à du «capital fixe collectif»).

Nous proposons, pour exprimer ce couplage PME/territoires, la notion de «Biens Territoriaux». De la même manière que les notions classiques de Biens Intermédiaires, de Biens d'Équipement et de Biens de Consommation rendent compte d'ensembles cohérents d'éléments concourant aux mêmes fonctions à l'intérieur du système productif (par exemple reproduction et développement du potentiel productif matériel pour les Biens d'Équipement), cette notion de Biens Territoriaux, en s'articulant transversalement aux trois autres, peut rendre compte d'un ensemble cohérent de ressources (PME, centres de recherche, potentiel de maind'oeuvre qualifiée, fonds d'aide à la création et à l'innovation, etc.) à la fois dans leurs inter-relations et dans leurs rapports à l'espace.

De la même manière également, l'accrochage renforcé des Biens Territoriaux à des logiques d'entreprises soumet ces «sous-systèmes» à des contraditions qui menacent sans cesse de faire éclater leur cohérence. Comme les PME, les territoires voient s'ouvrir la possibilité de conquérir des positions valorisantes, d'élargir leur marge d'autonomie, de s'imposer comme «points de passage obligé» (Callon, 1989). Mais comme les PME aussi, ils peuvent devenir «captifs» de ces mêmes positions, avec tous les risques induits par un profil trop exclusif type «zone de sous-traitance» (bassin d'Oyonnax pour le plastique, vallée de l'Arve pour le décolletage) ou type «zone d'innovation» (technopôles...)

Mais surtout, si les Biens Territoriaux sont cohérents entre eux, ils ne sont pas homogènes dans leur «construit»: le temps de l'émergence d'un potentiel de main-d'oeuvre qualifiée, d'un savoir-faire collectif, est bien supérieur au temps d'une «trajectoire» d'entreprise. Et la «norme internationale» de flexibilité, mobilité, adaptabilité, si prégnante pour les PME, ne peut être assimilée aussi aisément par des structures spatiales intrinsèquement porteuses de durée, de permanence, de stabilité des relations (Moulaert et Swyngedouw, 1988).

Même si les collectivités publiques ont justement pour rôle de gérer au mieux cette contradiction entre les visées à court terme du capital privé et les exigences territoriales s'inscrivant dans le plus long terme, les contraintes qui résultent de ce double rapport à l'espace et au temps risquent de rappeler très vite que le capital n'est pas seulement un «facteur opératoire». 
Les PME dans la nouvelle phase de régulation :

Tout dépend, en fait, de la logique véhiculée par les réseaux sur lesquels sont «branchés» ces Biens Territoriaux, c'est-à-dire de la logique de gestion par les firmes dominantes de leurs ressources externes. Pour les besoins de l'analyse, nous distinguerons deux positions «polaires» entre lesquelles la réalité s'oriente plus ou moins nettement, certaines firmes pouvant d'ailleurs combiner les deux.

a)

\section{La logique de l'«écrémage»}

C'est celle qui préside, pour de grandes firmes, à l'implantation, pour un temps déterminé, d' «usines tournevis» en fonction des seuls critères de coût de la main-d'oeuvre, d'accès aux réseaux de communications, de montant des subventions publiques. Ce type d'ancrage superficiel dans le territoire est exposé à des revirements brutaux de stratégie, comme en ont fait l'expérience récente les villes de Romans, dans la Drôme (l'entreprise italienne Maud Frizon a fermé en 1989 son usine de chaussures-150 personnes-implantée en 1986 et financée pour 8,7MF par la commune), ou de Stirling, en Écosse (l'informaticien américain WANG a décidé de fermer son usine-240 personnes -implantée en 1984 avec des aides publiques de $44 M F)$.

Cette logique peut se traduire aussi par la prédation pure et simple de savoirs et de savoir-faire repérés par les réseaux de «veille» de ces firmes (acquisition de procédés, main-mise sur des PME innovantes...), sans aucune préoccupation d'en renouveler le «stock».

b)

La logique de la «rente territoriale»:

Il nous paraît opportun de retenir la notion de rente car elle s'applique ici sous ses deux aspects les plus importants de rentabilité différentielle et de contrôle. Les firmes multinationales qui mettent cette logique en oeuvre cherchent en effet :

- à capter les effets utiles des «valeurs d'usage complexes» (Preteceille, 1974) que constituent les différentes ressources externes territorialisées (y compris les PME innovantes) et à les faire fonctionner en synergie avec leurs propres ressources internes. La distinction s'impose donc radicalement avec les transferts de profit «classiques» exercés aux dépens de sous-traitants ou de fournisseurs par le biais de la fixation des prix; s'il en résulte pour ces grandes firmes une élévation de rentabilité, c'est une élévation globale, dont les facteurs sont difficiles à individualiser, particulièrement ceux qui ont trait au nouveau type de rapport capital-travail et à la mobilisation spécifique de la maind'oeuvre qu'il induit. Il apparaît donc bien là une rente dont le différentiel se mesure par rapport aux firmes branchées sur des gisements moins riches en de telles ressources, mais surtout par rapportà celles qui en opèrent une valorisation moins efficace. 
- à s'assurer le contrôle sur l'accès à ces ressources mais aussi sur leur entretien, leur renouvellement, leur recomposition. A la différence d'une rente foncière, ce contrôle n'est lié ni à la propriété directe de ces ressources, ni à une position de monopole : même si, comme c'est souvent le cas dans cette option stratégique, il existe une présence concrète des firmes multinationales, leur contrôle s'exerce essentiellement sous une forme indirecte, par le biais d'un ou plusieurs réseaux, et met toujours soigneusement en avant l'aspect opératoire de toute implication du capital; par ailleurs, il peut être éventuellement «partagé» entre plusieurs firmes multinationales et fait appel de toutes façons au relais du capital public au travers surtout des Collectivités Territoriales.

Ce que nous entendons par «rente territoriale» prolonge ainsi la notion de «rente d'urbanisation» mise en oeuvre notamment par J.N. Thomas (1980) et qui traduit à la fois «l'évolution de l'ensemble des rapports sociaux vers la marchandise comme condition de la valorisation du capital» et un «transfert de rentabilité au profit des capitaux qui sont à même d'assurer une plus grande maîtrise collective de l'évolution sociale».

Une telle approche permet une lecture comparée des stratégies des différentes firmes multinationales selon qu'elles cherchent ou non à opérer une conjonction plus ou moins durable avec tout ou partie des biens territoriaux à leur disposition.

Ainsi, le succès économique du Land allemand de Bade-Wurtemberg nous semble tenir dans la forte et durable conjonction qui s'est établie entre ce territoire et des firmes aussi importantes que Siemens ou Mannesmann; elles conjuguent leur efforts avec celles de nombreuses $P M E$ (avec un appui actifdu Land et du Bund) pour tenir, notamment, l' ensemble de lafilière des systèmes de machines, de la conception à la formation des clients en passant par la fabrication de pratiquement tous les composants, mécaniques, hydrauliques, pneumatiques, électriques et électroniques et leur assemblage. La différence est flagrante avec la situation de Rhône-Alpes ou aucune grande firme, même publique, n'a daigné valoriser le riche potentiel existant dans cette même filière, avec des conséquences évidemment tout autre en matière de cohérence du tissu régional et d'autonomie du système productif national. ${ }^{13}$

Toujours est-il que pour les Collectivités Territoriales concernées qui ont de moins en moins la lecture d'ensemble de ce dispositif, une politique exclusive d'accès aux réseaux internationaux peut déboucher à terme sur la rupture des cohérences internes de leurs Biens Territoriaux et leur recomposition sélective et précaire sur quelques créneaux captifs de stratégies dont le contrôle échappe de plus en plus largement aux acteurs locaux.

Un tel risque est d'autant plus grand que la tendance actuelle est à la montée des grandes villes, appelées à être les «pivots du territoire» en s' intégrant aux noeuds

13 Nous travaillons actuellement dans le cadre d'un contrat de recherche «Plan Urbain» à l'étude des relations comparées de Hewlett-Packard et de Thomson avec le tissu socioéconomique de Rhône-Alpes. 
stratégiques des réseaux tout en contribuant à les renforcer (Perrat, 1990): «ll n'est pas neuf que les villes fonctionnent en réseau. Mais les réseaux hiérarchiques urbains d'échanges et d'influences décrits par nos grands historiens et géographes avaient en commun de couronner et de prolonger des hiérarchies territoriales $s_{\downarrow}$ Les réseaux nouveaux sont plus fluides, ils n'ont que faire des arrière-pays ou des zonages traditionnels. Et ils n'engagent souvent que des fractions urbaines sociales ou économiques marginales, bien que très dynamiques» (Beckouche, Savy et Veltz, 1988).

Il peut de cette façon se creuser des écarts d'autant plus importants qu'ils se combinent en amplification de tendances :

- au sein des tissus régionaux et nationaux, entre les zones «bien» et «mal» placées sur la carte européenne (et mondiale) de ce nouveau «territoire des intensités». Cette carte, dans toutes ses versions connues, n'inscrit la France qu'à la marge; encore faut-il pour cela que le passage des grands flux Nord-Sud, non encore définitivement ancrés, ne glisse pas trop à l'Est. Même en RhôneAlpes, pourtant une des régions les plus proches des grandes densités européennes, la frontière des opportunités ne dessine guère plus qu' un «triangle Lyon-Grenoble-Genève», et M. Bonneville peut parler à juste titre de «l'Ouest débranchéx.

- au coeur même des villes-pôles, fussent-elles des «Eurocités», entre des structures, des entreprises et des hommes pouvant bénéficier des fonctions de la «centralité» et ceux qui en sont exclus. Avoir accès à cette «centralité», en effet, est moins affaire de proximité spatiale que de proximité économique, technologique sociale ou culturelle (Bonneville, 1989).

\section{Conclusion}

Les PME (ou du moins un nombre important d'entre elles) évoluent vers des formes aux frontières moins définies, se rapprochant de la structure en réseau initiée par les grandes firmes, et surtout vers des modes de propriété et de fonctionnement qui tendent à établir un rapport du travail au capital plus instrumental, estompant l'aspect rapport social conflictuel.

Cette évolution permet - en même temps qu'elle est induite par - un positionnement nouveau de ces PME dans le système productif et les processus de production, dans la mise en oeuvre des ressources internes comme dans l'accès aux ressources externes, même si ce dernier appelle le relais des institutions publiques et des Collectivités Territoriales. Ces positions sont plus valorisantes, mais sont à la fois porteuses de plus d'autonomie et d'une insertion accrue dans les réseaux contrôlés par les firmes multinationales.

En termes de régulation, il y a là tous les éléments, au moins potentiellement, d'une efficacité supérieure : 
- par une meilleure mobilisation des ressources, particulièrement des ressources humaines;

- par l'ouverture, au travers du phénomène de «partenariat», d'un processus de dépassement des relations strictement concurrentielles et strictement marchandes (Tetra, 1990);

- par la réduction de la fracture entre PME et grandes firmes et par l'implication plus grande de ces dernières dans les «territoires», en rupture avec leur pratique antérieure d'exploitation plus extensive de l'«espace».

Mais les limites à l'expression maximale de cette efficacité tiennent justement à cette implication plus poussée des firmes multinationales dans la gestion de l'ensemble des «biens territoritaux» dont le devenir se trouve, du coup, plus fortement dépendant de leur stratégie et de la logique qui l'imprègne, selon qu'elle penche plutôt vers l'écrémage ou plutôt vers une valorisation des potentiels matériels et humains. La régulation qui tente ainsi de se constituer risque donc de se heurter à des contre-tendances qui en réduisent à la fois l'efficacité et le champ d'application:

- une pénétration très sélective, fractionnée et limitée dans les processus productifs d'un mode de mobilisation supérieure de la force de travail, alors que monte l'exigence de sa mise en oeuvre plus collective et plus large;

- un «rappel» constant à la prédominance de la concurrence sur la coopération, du marchand sur le non marchand;

- une valorisation des Biens Territoriaux elle aussi sélective, hiérarchisée et porteuse d'exclusions introduisant forcément des effets en retour négatifs, ne serait-ce qu'en termes de coûts;

- une mise en rapport au temps de ces ressources internes et externes qui traduit souvent «flexibilité» par «précarité» et qui entre ainsi en conflit avec la stabilité nécessaire à l'émergence de synergies et de «fertitilisations croisées».

Se trouve ainsi posée une question qui mérite débat : au regard du développement d'un système productif, quelle que soit son échelle, un réseau peut-il constituer durablement un niveau de cohérence, une instance de régulation ?

Un psychologue travaillant pour le compte d'un Institut de Développement irlandais écrivait récemment : «Il y a un tas d'énergie et de capacité chez les gens encore inutilisés. Nous avons besoin d'une réflexion nouvelle pour saisir cette énergie et cette capacité» ${ }^{14}$. Nous pensons que c'est effectivement là un des deux principaux problèmes qui se posent à la nouvelle régulation, avec celui de réussir à construire les bons niveaux de cohérence (Europe ? Nation ? Région ? Réseaux de villes ? Réseaux de firmes?...). Mais pour nous le défi fondamental à relever pour avancer en ce sens est justement de parvenir à mobiliser cette énergie et cettecapacité, dans l'entreprise comme dans les territoires, sans ni la «brider», ni la «capturer».

14 «There is a lot of energy and capacity in people, as yet unharnessed. We need fresh thinking on how to capture that energy and capacity» (T.S.D., 1989). 


\section{BIBLIOGRAPHIE}

Aglietta, M. et Brender, K, (1984), Les métamorphoses de la société salariale : la France en projet, Paris, Calmann-Levy, 1984.

Beckouche, P., Savy, M. et Veltz, P., (1988), «Nouvelle économie, nouveaux territoires», Lettre de la Datar, $\mathrm{n}^{\circ} 3$, juin 1988.

Bellet, M., (1987), «Régulation, rapport salarial, critères de gestion [...] éléments pour une confrontation", La transformation du rapport salarial, Lyon, A.E.H.-P.U.L.

Bonneville, M., (1989), «Lecentre et ses périphéries dans la prospective de Lyon 2010», Revue de Géographie de Lyon, vol. 64, $\mathrm{n}^{\circ} 1$.

Boyer, R., (1985), «Les approches en termes de régulation : présentation et problèmes de méthodes», Do. CEPREMAP, juillet.

Callon, M., (1989), «L'économie des réseaux technico-économiques», Paris, École des Mines, document provisoire.

Christiansen, P.R., (1988), «Flexibilité de l'entreprise et réseaux régionaux», dans Industrialisationflexible, perspectives françaises et danoises, Grenoble, Cahiers de l'IREP$\mathrm{D}-\mathrm{n}^{\circ} 12$,

Delapierre, M. et Michalet, C.A., (1989), «Vers un changement des structures des multinationales : le principe d'internalisation en question", Revue d' économie industrielle, $\mathrm{n}^{\circ} 47$, ler trimestre.

De Montmorillon, B., (1989), «Vers une reformulation de la théorie du groupe», Revue $d^{\prime}$ économie industrielle, $\mathrm{n}^{\circ} 47$, ler trimestre.

Ganne, B., (1988), «La mutation industrielle des PME annonéennes», Milieu industriel et systèmes industriels locaux, Lyon, Groupe Lyonnais de Sociologie Industrielle, doc. $n^{\circ} 1$.

Le Bas, C., (1987), La transformation du rapport salarial, présentation, Lyon, A.E.H.-P.U.L.

Lecler, Y., (1989), «Le transfert de compétences entre grandes entreprises donneuses d'ordres et PMI sous-traitantes : analyse du partenariat à la lumière de l'exemple japonais, dans J.H. Jacot et J. Saglio, «Dynamique de développement des PME : transfert de compétences, réseaux de PME et politiques de formation», rapport intermédiaire au PPSH, Lyon, juin.

Monsted, M., (1988), «Industrialisation flexible et qualification dans les petites entreprises de la métallurgie danoise», dans Industrialisation flexible, perspectives françaises et danoises, Grenoble, Cahiers de l'IREP-D n ${ }^{\circ} 12$.

Moulaert, F. et Swyngedow, E., (1988), «Développement régional et géographie de la production flexible», Cahiers Lillois d'Économie et de Sociologie, $\mathrm{n}^{\circ} 11$.

Perrat, J., (1988), «Gestion des externalités et nouvel usage des formes Région et Europe», Revue d'Économie Régionale et Urbaine, 5.

Perrat, J., (1990), «Le réseau des villes : des territoires régionaux à l'espace européen?», communication au colloque de l'A.S.R.D.L.F., «Mondialisation de l'économie et développement des territoires», St-Étienne, du 3 au 5 septembre 1990.

Preteceille, E., (1974), «La planification urbaine. Les contradictions de l'urbanisation capitaliste», Économie et politique, $\mathrm{n}^{\circ} 236$, mars.

Raveyre, M.F., (1988), «Jeux de miroirs. L'aide de S.G.D. aux PME», Lyon, GLYSI, doc. $n^{\circ} 2$.

Tetra, (1990), colloque La PME objet de recherche pertinent?, Lyon, 30-31 mai 1990, organisé par le Groupe Technologie Emploi Travail Rhône-Alpes réunissant plusieurs équipes de recherche de Lyon, Saint-Étienne et Grenoble.

Thomas, J.N., (1980), La rente d' urbanisation, Lyon, P.U.L.

T.S.D. (Technopôle Service Développement), (1989), «Pour une stratégie régionale de soutien à l' innovation et au développement technologique des PMI en Rhône-Alpes», Sophia Antipolis, annexes p. 141. 